\title{
Topological interface physics of defects and textures in spinor Bose-Einstein condensates
}

\author{
Magnus O. Borgh* and Janne Ruostekoski ${ }^{\dagger}$ \\ School of Mathematics, University of Southampton, SO17 1BJ Southampton, United Kingdom
}

(Received 10 December 2012; published 19 March 2013)

\begin{abstract}
We provide a detailed description of our previously proposed scheme for topological interface engineering with constructed defects and textures perforating across coherent interfaces between different broken symmetries [Borgh and Ruostekoski, Phys. Rev. Lett. 109, 015302 (2012)]. We consider a spin-1 Bose-Einstein condensate, in which polar and ferromagnetic phases are prepared in spatially separated regions. We show that a stable coherent interface is established between the two phases, allowing defects of different topology to connect continuously across the boundary. We provide analytic constructions of interface-crossing defect solutions that could be experimentally phase imprinted using existing technology. By numerically minimizing the energy, we calculate the core structures of interface-crossing defect configurations. We demonstrate nontrivial core deformations to considerably more complex structures, such as the formation of an arch-shaped half-quantum line defect, an Alice arch, at the interface, with the topological charge of a point defect, whose emergence may be understood by the "hairy ball" theorem. Another example of an energetically stable object is the connection of a coreless vortex to a pair of half-quantum vortices. We show that rotation leads to spontaneous nucleation of defects in which a coreless vortex continuously transforms to a half-quantum vortex across the interface.
\end{abstract}

DOI: 10.1103/PhysRevA.87.033617 PACS number(s): 03.75.Lm, 03.75.Mn, 67.85.Fg, 11.27.+d

\section{INTRODUCTION}

In systems described by an order parameter, for example superfluid liquid helium, liquid crystals, and Bose-Einstein condensates (BECs), the symmetry properties of this order parameter determine the topological properties of defects and textures [1]. Systems with vector order parameters, such as superfluid liquid ${ }^{3} \mathrm{He}$ [2] and spinor BECs [3-5], may exist in several distinct phases. Each phase corresponds to a different ground-state manifold of energetically degenerate and physically distinguishable states, resulting in different order-parameter symmetries. When two phases coexist in spatially separated regions in the same medium, topological defects cannot penetrate the boundary unchanged, but must either terminate at the interface or connect nontrivially to an object on the other side. Topological interfaces appear as important objects in seemingly distant areas of physics. For example, it has been proposed that a series of symmetry breakings in the early universe leads to the formation of cosmic strings that terminate on boundaries between regions of different vacua $[6,7]$. Complex interface physics also arises in string theory from collisions between branes during inflation $[8,9]$, in condensed-matter theory in exotic superconductivity [10], and in superfluid liquid ${ }^{3} \mathrm{He}$ when a magnetic-field gradient causes $A$ and $B$ phases to coexist, resulting in the possibility of nontrivial defects at the phase boundary [11-14]. Parallels between defects in superfluids and objects in cosmology [7] led to the suggestion of using superfluid systems to study analogs of cosmological phenomena in the laboratory $[12,15]$, including defect formation in phase transitions [16-18], analogs of brane annihilation [14,19], and structures similar to cosmic vortons [20-22].

Here we present a detailed description of our proposal for an experimentally feasible scheme to study topological

\footnotetext{
*M.O.Borgh@soton.ac.uk

†janne@soton.ac.uk
}

interface physics in a gas of ultracold atoms with spin degree of freedom [23]. A stable, coherent interface between two ground-state manifolds of different broken symmetries may be prepared by local manipulation of the interaction strengths of different scattering channels of the atoms. The proposed technique can be used to realize, e.g., two different groundstate manifolds simultaneously in spatially separate regions. Defects and textures crossing the interface, and connecting objects of different topologies, can be created by controlled phase imprinting of elementary vortex and soliton structures or by rotating the gas. Under dissipation the phase-imprinted defect configurations relax to more complex objects for which the continuous spinor wave function interpolates smoothly across the topological interface.

As an example we consider defects and textures crossing the boundary between polar and ferromagnetic (FM) regions in a spin-1 BEC and show that a coherent interface is established within a continuous condensate wave function. We analytically construct prototype spinor wave functions representing interface-crossing defect structures, and by numerically minimizing their energy, we evaluate the configurations that emerge as a result of energy dissipation in spin-1 BECs. The simulations demonstrate nontrivial core deformations and defect structures. We characterize the defect cores, analyze the energetic stability of defect solutions crossing the interface, and explain how the defect-carrying condensate wave function continuously interpolates across the interface. We also demonstrate nucleation of interface-crossing defects consisting of a coreless vortex that connects to a half-quantum vortex.

A spinor BEC [3-5] is created in an all-optical trap so that the spin degree of freedom of the atoms is not frozen out by magnetic fields. Spin rotations then combine with the condensate phase to form a large set of physically distinguishable states. Because also the contact interaction between the atoms in the condensate becomes spin dependent, energetically degenerate subsets depend on the strength and sign of the spin-dependent contributions. Hence the spinor BEC exhibits a rich diagram of phases with different broken 
order-parameter symmetries [24-30] as a function of the interaction strengths. This is similar to superfluid liquid ${ }^{3} \mathrm{He}$ where nonzero spin and orbital angular momenta of the Cooper pairs combine to form phases with different order-parameter symmetries [2] supporting a variety of defects and textures [31].

Modern techniques used in experiments with ultracold atoms provide tools for unprecedented control over system parameters and for accurate measurements, including the possibility for in situ observation of vortices in spinor BECs. As there has been considerable interest in the studies of the stability properties of field-theoretical solitons in various physical systems [32-35], it is therefore not surprising that this is also followed by an accelerating theoretical interest in a variety of stable and metastable objects in multicomponent atomic BECs. Perhaps the simplest of such structures where the multicomponent nature of BECs plays an important role are one-dimensional (1D) vector solitons [36-43], typically consisting of stable combinations of dark and bright solitons in different condensate components. Higher-dimensional defects and textures include vortex sheets [44] and 3D particlelike solitons [20-22,45-47] in two-component (pseudospin-1/2) condensates, as well as a rich phenomenology of defects and textures in spin-1 [23,24,26,48-63], spin-2 [64-66], and spin-3 [30,67] BECs. Interface physics has been studied in two-component BEC systems, for example, in the context of vortex bifurcation at energetically established interfaces in the phase-separation regime [68,69] and interface collisions [19]. There is a rapid parallel experimental development, exemplified by preparation of coreless vortices and related textures [70-73], as well as observations of singular vortices produced in phase transitions [74], and of spin-texture formation [75-77]. Furthermore, trapping of ultracold atoms in artificial gauge-field potentials [78] was recently realized experimentally $[79,80]$. This presents intriguing possibilities for the stability studies of defects and textures, including those of particlelike solitons [47].

Our study of topological interface engineering is organized as follows: In Sec. II, we first give a brief overview of the standard mean-field theory of the spin-1 BEC and then proceed to give a more detailed presentation of the topology and basic defects of the FM and polar phases. In Sec. III we discuss how an interface between FM and polar regions can be created, and then identify and explicitly construct interface-crossing defect solutions. We proceed to minimize the energy of the defect solutions in Sec. IV and describe the emerging structures of the defect core and the energetic stability of the defects. We explicitly demonstrate continuity of the spinor wave function across the stable interface. We summarize our findings in Sec. V.

\section{SPIN-1 BEC}

\section{A. Mean-field theory of spin-1 BEC}

We consider a trapped spin-1 atomic BEC confined in an all-optical, harmonic trap. We may then employ the classic Gross-Pitaevskii mean-field theory describing a spatially inhomogeneous macroscopic condensate wave function $\Psi(\mathbf{r})$. Since we are considering spin-1 atoms, $\Psi(\mathbf{r})$ can be written in terms of the density of atoms $n(\mathbf{r})$ and a normalized, three-component spinor $\zeta(\mathbf{r})$ in the basis of spin projection onto the $z$ axis as

$$
\Psi(\mathbf{r})=\sqrt{n(\mathbf{r})} \zeta(\mathbf{r})=\sqrt{n(\mathbf{r})}\left(\begin{array}{c}
\zeta_{+}(\mathbf{r}) \\
\zeta_{0}(\mathbf{r}) \\
\zeta_{-}(\mathbf{r})
\end{array}\right), \quad \zeta^{\dagger} \zeta=1 .
$$

The mean-field Hamiltonian density then reads [24,25,81]

$$
\begin{aligned}
\mathcal{H}= & \frac{\hbar^{2}}{2 m}|\nabla \Psi|^{2}+V(\mathbf{r}) n+\frac{c_{0}}{2} n^{2}+\frac{c_{2}}{2} n^{2}|\langle\hat{\mathbf{F}}\rangle|^{2} \\
& +g_{1} n\langle\mathbf{B} \cdot \hat{\mathbf{F}}\rangle+g_{2} n\left\langle(\mathbf{B} \cdot \hat{\mathbf{F}})^{2}\right\rangle,
\end{aligned}
$$

where $V(\mathbf{r})$ is an external trapping potential and $m$ is the atomic mass. In this work we consider the atoms trapped in a slightly elongated potential, so that

$$
V(\mathbf{r})=\frac{1}{2} m \omega^{2}\left(x^{2}+y^{2}+\frac{z^{2}}{4}\right) .
$$

The spin operator $\hat{\mathbf{F}}$ is given by a vector of spin-1 Pauli matrices. Its expectation value $\langle\hat{\mathbf{F}}\rangle=\zeta_{\alpha}^{\dagger} \hat{\mathbf{F}}_{\alpha \beta} \zeta_{\beta}$ is the local spin vector. A weak external magnetic field may be imposed, in which case linear and quadratic Zeeman shifts as described by the last two terms will arise. Most of our numerical results correspond to cases for which the Zeeman splitting is assumed to be negligible. We note, however, that all our results remain qualitatively the same in the presence of weak Zeeman splitting energy.

We also investigate the configurations of defects and textures in a rotating trap. In that case we minimize the free energy in a rotating frame, corresponding to

$$
\begin{aligned}
H^{\prime} & =H-\langle\Omega \cdot \hat{\mathbf{L}}\rangle \\
& =\int d^{3} r\left[\mathcal{H}(\mathbf{r})+i \hbar \Omega \Psi^{\dagger}(\mathbf{r})\left(x \frac{\partial}{\partial y}-y \frac{\partial}{\partial x}\right) \Psi(\mathbf{r})\right],
\end{aligned}
$$

where we have assumed the axis of rotation defined by $\Omega$ to be along the positive $z$ axis, and $\hat{\mathbf{L}}$ denotes the angular-momentum operator.

The two interaction terms in Eq. (2) arise from the fact that the spins of two colliding spin-1 atoms may combine to either 0 or 2 . There are therefore two $s$-wave scattering channels, with scattering lengths $a_{0}$ and $a_{2}$, contributing to the contact interaction between the atoms in the condensate. Standard angular-momentum algebra [81] separates the interaction energy into one spin-independent contribution and one term that depends on the magnitude of the spin. The strengths of the spin-independent and spin-dependent interaction terms are then given by

$$
c_{0}=\frac{4 \pi \hbar^{2}\left(2 a_{2}+a_{0}\right)}{3 m}, \quad c_{2}=\frac{4 \pi \hbar^{2}\left(a_{2}-a_{0}\right)}{3 m},
$$

respectively. Additional magnetic dipole-dipole interactions that may influence the spin textures $[62,75,82]$ are neglected here.

The sign of $c_{2}$, the strength of the spin-dependent interaction, determines the magnitude of the spin vector in a uniform ground state, leading to the two topologically distinct phases of the spin-1 BEC. If $c_{2}<0$, energy minimization favors maximized spin magnitude $|\langle\hat{\mathbf{F}}\rangle|=1$ in the FM phase. This 
is the case for ${ }^{87} \mathrm{Rb}$ where $c_{0} / c_{2} \simeq-216$ [83]. Conversely, if $c_{2}>0$, as for ${ }^{23} \mathrm{Na}$ with $c_{0} / c_{2} \simeq 31$ [84], $|\langle\hat{\mathbf{F}}\rangle|=0$ is favored in the polar phase. The two phases are described by fundamentally different order parameters, supporting different families of defects, which we will discuss in some detail below.

Characteristic length scales arise from the interaction terms. The spin-independent interaction defines the usual density healing length

$$
\xi_{n}=\frac{1}{\sqrt{8 \pi c_{0} n}}
$$

which describes the length scale over which the atom density $n(\mathbf{r})$ heals around a local density depletion. In addition, the spin-dependent interaction gives rise to a spin healing length

$$
\xi_{F}=\frac{1}{\sqrt{8 \pi\left|c_{2}\right| n}},
$$

defining the distance over which $|\langle\hat{\mathbf{F}}(\mathbf{r})\rangle|$ heals as the order parameter is excited out of its ground-state manifold. That situation arises in two cases of importance for the analysis presented in this article. First, the core of a singular vortex in one phase may fill with atoms such that the atoms at the singularity exhibit the opposite phase. This can happen since the singularity of the spinor order parameter may be accommodated either by forcing the density to zero or by requiring that the wave function become orthogonal to the ground-state manifold (meaning locally perturbing $|\langle\hat{\mathbf{F}}\rangle|$ ) $[55,63]$. The size of the filled vortex core is then determined by $\xi_{F}$. Second, we are interested here in the interface between polar and FM regions. For the condensate wave function to interpolate between the two manifolds, the spin magnitude must leave its ground-state value close to the interface, which will therefore acquire a width determined by $\xi_{F}$.

\section{B. Ground-state manifolds and basic defects}

The order-parameter manifold is the set of energetically degenerate, physically distinguishable states. In the condensation transition, this symmetry is spontaneously broken, and this broken symmetry determines the topologically distinct families of defects. The FM and polar phases of the spin-1 BEC are described by very different order-parameter manifolds, leading to dramatically different possible vortex states. Before discussing the interface between FM and polar regions in the next section, we here give an overview of the families of defects in the purely FM or purely polar BEC.

\section{FM phase}

If $c_{2}<0$ in Eq. (2), the spin-dependent interaction will favor a state that maximizes the magnitude of the spin everywhere, such that $|\langle\hat{\mathbf{F}}\rangle|=1$. A representative FM spinor is given by $\zeta=(1,0,0)^{T}$, such that the spin vector is parallel with the $z$ axis. From this representative spinor, a general FM spinor may be constructed by a 3D spin rotation,

$$
U(\alpha, \beta, \gamma)=\exp \left(-i F_{z} \alpha\right) \exp \left(-i F_{y} \beta\right) \exp \left(-i F_{z} \gamma\right)
$$

defined by three Euler angles, together with a condensate phase $\phi$, as

$\zeta^{\mathrm{f}}=e^{i \phi} U(\alpha, \beta, \gamma)\left(\begin{array}{l}1 \\ 0 \\ 0\end{array}\right)=\frac{e^{-i \gamma^{\prime}}}{\sqrt{2}}\left(\begin{array}{c}\sqrt{2} e^{-i \alpha} \cos ^{2} \frac{\beta}{2} \\ \sin \beta \\ \sqrt{2} e^{i \alpha} \sin ^{2} \frac{\beta}{2}\end{array}\right)$,

where the condensate phase is absorbed in the third Euler angle: $\gamma^{\prime}=\gamma-\phi$. Any FM spinor is thus described by some particular choice for $\left(\alpha, \beta, \gamma^{\prime}\right)$. Therefore the broken symmetry of the ground-state manifold is represented by the group of 3D rotations $\mathrm{SO}(3)$. The spin vector is given by the Euler angles as $\langle\hat{\mathbf{F}}\rangle=(\cos \alpha \sin \beta, \sin \alpha \sin \beta, \cos \beta)$.

Topological stability of line defects can be characterized by studying closed contours around the defect line and the mapping of these contours into order-parameter space [1]. If the image in order-parameter space of a closed loop encircling a line defect can be contracted to a point, the defect is not topologically stable. The order-parameter space of a FM spin-1 BEC, $\mathrm{SO}(3)$, can be represented geometrically as $S^{3}$ (the unit sphere in four dimensions) with diametrically opposite points identified. If a closed contour connects such identified points more than once, any pair of such connections can be eliminated by continuous deformation of the contour. Therefore any contour with an even number of connections can be contracted to a point, whereas a contour with an odd number of connections can be deformed into a contour with just one connection. Hence we have only two distinct classes of vortices: singly quantized, singular vortices that correspond to noncontractible loops, and nonsingular, coreless vortices representing contractible loops. All other vortices can be transformed to either one of these by local deformations of the order parameter. Mathematically, these equivalence classes are characterized by the two elements of the first homotopy group, $\pi_{1}[\mathrm{SO}(3)]=\mathbb{Z}_{2}$.

The simplest representative of the class of singular line defects is constructed as a $2 \pi$ winding of the condensate phase such that $\gamma^{\prime}=-\varphi$ in Eq. (9), where $\varphi$ is the azimuthal angle. This results in a line singularity oriented along the $z$ axis. The spin texture is uniform, such that $\alpha$ and $\beta$ are arbitrary but constant. The vortex is then described by the spinor

$$
\zeta^{\mathrm{s}}(\mathbf{r})=\frac{e^{i \varphi}}{\sqrt{2}}\left(\begin{array}{c}
\sqrt{2} e^{-i \alpha} \cos ^{2} \frac{\beta}{2} \\
\sin \beta \\
\sqrt{2} e^{i \alpha} \sin ^{2} \frac{\beta}{2}
\end{array}\right) .
$$

From $\zeta^{\text {s }}$ other vortices in the same equivalence class can be formed by local spin rotations. For example, we may rotate the spin vector such that at each point it points radially away from the vortex line, as illustrated in Fig. 1(a). This vortex corresponds to the choices $\alpha=\varphi, \beta=\pi / 2$, and $\gamma^{\prime}=0$ in Eq. (9), and is described by

$$
\zeta^{\mathrm{sv}}=\frac{1}{2}\left(\begin{array}{c}
e^{-i \varphi} \\
\sqrt{2} \\
e^{i \varphi}
\end{array}\right)
$$

This singular spin vortex $[24,25]$ illustrates another important aspect of the FM phase: circulation alone is not quantized. The 


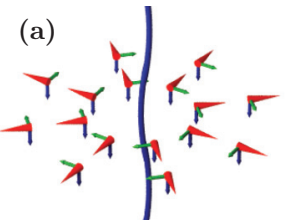

(c)
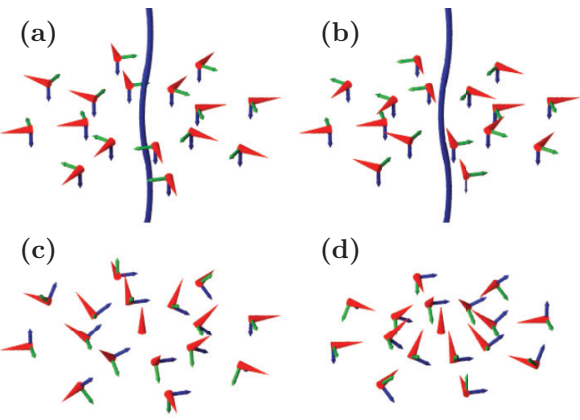

(d)

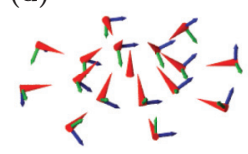

FIG. 1. (Color online) Nontrivial vortices in the FM phase. (a) A radial disgyration of the spin vector (red cones) around the singular core represents a spin vortex. This vortex carries a spin current but no mass circulation, a manifestation of the nonquantization of circulation alone in the FM phase. (b) All vortices in the class of singly quantized, singular vortices can be deformed into each other by local spin rotations. For example, it is possible to form a spin vortex with a cross-disgyration of the spin vector. (c) In addition to the one class of singular vortices, the FM phase also supports nontrivial, nonsingular vortices. In a coreless vortex the order parameter remains nonsingular everywhere in the vortex structure. The characteristic fountain structure of the spin is formed by a rotation of the spin vector around the vortex line, together with a winding of the condensate phase, corresponding to spin rotations about the local spin vector [indicated by the orthogonal green and blue (light and dark gray) vectors]. (d) Like the class of singular line defects, members of the family of nonsingular vortices are related by local deformations of the order parameter allowing different vortex configurations.

superfluid velocity in the FM phase [24],

$$
\mathbf{v}=-\frac{\hbar}{m}\left(\nabla \gamma^{\prime}+\cos \beta \nabla \alpha\right),
$$

vanishes when $\gamma^{\prime}=0$ and $\beta=\pi / 2$. Therefore the circulation is in fact zero in $\zeta^{\text {sv }}$, but it does carry a nonvanishing spin current around a singularity of the FM spin vector, whose structure is similar to an analogous vortex with a radial disgyration of the angular momentum vector in ${ }^{3} \mathrm{He}$. Further local spin rotations yield other singular vortices with different spin structures, such as the cross disgyration shown in Fig. 1(b) or a tangential disgyration with $\langle\hat{\mathbf{F}}\rangle=\hat{\boldsymbol{\varphi}}$.

A striking manifestation of the nonquantization of circulation in the FM phase is the formation of a nonsingular coreless vortex. This can be constructed as a combined rotation of the spin vector and the condensate phase [Fig. 1(c)]: $\alpha=-\gamma^{\prime}=\varphi$, yielding the spinor

$$
\zeta^{\mathrm{cl}}(\mathbf{r})=\frac{1}{\sqrt{2}}\left(\begin{array}{c}
\sqrt{2} \cos ^{2} \frac{\beta(\rho)}{2} \\
e^{i \varphi} \sin \beta(\rho) \\
\sqrt{2} e^{2 i \varphi} \sin ^{2} \frac{\beta(\rho)}{2}
\end{array}\right),
$$

where the Euler angle $\beta$ is now a function of the radial distance $\rho=\sqrt{x^{2}+y^{2}}$, such that $\beta \rightarrow 0$ as $\rho \rightarrow 0$, keeping the spin texture continuous. The superfluid velocity, Eq. (12), becomes

$$
\mathbf{v}^{\mathrm{cl}}=\frac{\hbar}{m \rho}(1-\cos \beta) \hat{\boldsymbol{\varphi}},
$$

and increases smoothly from zero at $\rho=0$ as $\beta$ increases away from the vortex, the spin vector forming a fountainlike

texture. The coreless vortex in the FM phase of a spin-1 BEC is analogous to the Anderson-Toulouse and Mermin-Ho vortices in superfluid ${ }^{3} \mathrm{He}[85,86]$, which differ by the boundary condition imposed on the angular momentum vector at the container wall. In the BEC there is no hard container wall, and the amount by which $\beta$ turns from the vortex line to the edge of the cloud is determined by the rotation of the trap, causing the total angular momentum to vary smoothly with rotation.

The coreless vortex can be continuously transformed into other members of the class of nonsingular vortices, including the vortex-free state, by purely local operations. The continuous deformation is a striking consequence of the two-element character of the fundamental group of the $\mathrm{SO}(3)$ order-parameter space: the doubly quantized vortex belongs to the same topological class as the nonsingular vortices and the vortex-free state, and can be continuously unwound, if the orientation of the spin texture is not fixed outside the structure. Another nontrivial nonsingular vortex with continuous spin textures is displayed in Fig. 1(d).

We have now identified two topologically distinct classes of vortices that can both carry mass and spin circulation in the FM spin-1 BEC. A similar situation applies in the $A$ phase of ${ }^{3} \mathrm{He}$. There the singular vortex has the lower energy, but the energy barrier for nucleation is lower for the nonsingular vortex [87]. In a rotating FM spin-1 BEC, the coreless vortex has the lower energy and the lower nucleation barrier, and consequently the ground state is made up of nonsingular coreless vortices for sufficiently rapid rotation [53,54,57,58,61]. However, it is also possible to form a singly quantized, singular vortex $[51,52]$, which despite not being the lowest-energy state at any frequency of rotation can nevertheless be energetically stable as a local energy minimum [63].

So far we have considered line defects, classified by the first homotopy group $\pi_{1}$. Point defects-monopoles-are analogously classified by the second homotopy group $\pi_{2}$. For the FM order-parameter manifold, the second homotopy group is the trivial group, $\pi_{2}[\mathrm{SO}(3)]=0$, indicating that the FM phase does not strictly speaking support point defects. However, it is possible to form a spinor with a monopole structure of the spin vector (a radial hedgehog) as the termination of a doubly quantized vortex [56] (Fig. 2). This is the analog of the Dirac monopole in quantum field theory, and the doubly quantized vortex line is called the Dirac string. The corresponding spinor is written by choosing $\alpha=\gamma^{\prime}=\varphi$ and $\beta=\theta$ (where $\theta$ and $\varphi$ are the polar and azimuthal angles,

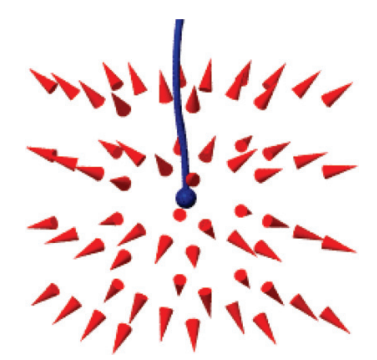

FIG. 2. (Color online) An analog of the field-theoretical Dirac monopole can be constructed in the FM phase of the spin-1 BEC. The radial hedgehog monopole structure of the spin vector (cones) appears as the termination of a doubly quantized, singular vortex line. 
respectively) to form

$$
\zeta^{\mathrm{D}}=\frac{1}{\sqrt{2}}\left(\begin{array}{c}
\sqrt{2} e^{-2 i \varphi} \cos ^{2} \frac{\theta}{2} \\
e^{-i \varphi} \sin \theta \\
\sqrt{2} \sin ^{2} \frac{\theta}{2}
\end{array}\right) .
$$

This Dirac monopole can be continuously deformed into the spin structure of the coreless vortex [56].

\section{Polar phase}

We next consider $c_{2}>0$ in Eq. (2), in which case the spindependent interaction favors a state with $|\langle\hat{\mathbf{F}}\rangle|=0$. A simple representative polar spinor fulfilling this requirement is $\zeta=$ $(0,1,0)^{T}$. As for the FM phase, the general polar spinor is found by applying a 3D spin rotation $U(\alpha, \beta, \gamma)$ together with a condensate phase $\phi$ :

$$
\zeta^{\mathrm{p}}=e^{i \phi} U(\alpha, \beta, \gamma)\left(\begin{array}{l}
0 \\
1 \\
0
\end{array}\right)=\frac{e^{i \phi}}{\sqrt{2}}\left(\begin{array}{c}
-e^{-i \alpha} \sin \beta \\
\sqrt{2} \cos \beta \\
e^{i \alpha} \sin \beta
\end{array}\right) .
$$

We now make the important observation that the unit vector $\hat{\mathbf{d}}=(\cos \alpha \sin \beta, \sin \alpha \sin \beta, \cos \beta)$ defines the local direction of macroscopic condensate spin quantization. This allows us to rewrite $\zeta^{\mathrm{p}}$ in terms of this vector as [55]

$$
\zeta^{\mathrm{p}}=\frac{e^{i \phi}}{\sqrt{2}}\left(\begin{array}{c}
-d_{x}+i d_{y} \\
\sqrt{2} d_{z} \\
d_{x}+i d_{y}
\end{array}\right) .
$$

The condensate phase $\phi$, which takes values on a unit circle, and the unit vector $\hat{\mathbf{d}}$, taking values on a sphere, thus together fully specify the order parameter in the polar phase. Note, however, that $\zeta^{\mathrm{p}}(\phi, \hat{\mathbf{d}})=\zeta^{\mathrm{p}}(\phi+\pi,-\hat{\mathbf{d}})$. These two states must be identified in order to avoid double counting. The order parameter space therefore becomes $\left[\mathrm{U}(1) \times S^{2}\right] / \mathbb{Z}_{2}$, where the factorization by the two-element group $\mathbb{Z}_{2}$ results from the identification. The vector $\hat{\mathbf{d}}$ should thus be taken to be unoriented and defines a nematic axis [26], and the order-parameter is correspondingly said to exhibit nematic order, which leads to parallels with the $A$ phase of superfluid ${ }^{3} \mathrm{He}$.

A simple singly quantized vortex can again be constructed as a $2 \pi$ winding of the condensate phase, keeping $\hat{\mathbf{d}}$ uniform (choosing $\alpha$ and $\beta$ to be constants):

$$
\zeta^{1}=\frac{e^{i \varphi}}{\sqrt{2}}\left(\begin{array}{c}
-e^{-i \alpha} \sin \beta \\
\sqrt{2} \cos \beta \\
e^{i \alpha} \sin \beta
\end{array}\right) .
$$

In the polar phase the superfluid velocity is [4]

$$
\mathbf{v}=\frac{\hbar}{m} \nabla \phi .
$$

We observe that $\mathbf{v}$ depends only on the gradient of the condensate phase, and is independent of $\hat{\mathbf{d}}$. This means that another singly quantized vortex, with the same circulation as that described by Eq. (18), can be formed by allowing $\hat{\mathbf{d}}$ to wind by $2 \pi$ (thus preserving single-valuedness of the order parameter) in addition to the winding of the condensate phase.
This is achieved by choosing $\alpha=\phi=\varphi$ in Eq. (16), yielding the spinor

$$
\zeta^{1^{\prime}}=\frac{1}{\sqrt{2}}\left(\begin{array}{c}
-\sin \beta \\
\sqrt{2} e^{i \varphi} \cos \beta \\
e^{2 i \varphi} \sin \beta
\end{array}\right)
$$

One can further show from Eq. (19) that circulation is quantized in the polar phase. However, due to the nematic order, the smallest circulation possible is half that of a singly quantized vortex. The equivalence $\zeta^{\mathrm{p}}(\phi, \hat{\mathbf{d}})=\zeta^{\mathrm{p}}(\phi+\pi,-\hat{\mathbf{d}})$ implies that we can allow the condensate phase to wind by $\pi$ along a loop encircling the vortex and still preserve single-valuedness of the the order parameter by a simultaneous $\hat{\mathbf{d}} \rightarrow-\hat{\mathbf{d}}$ winding of the nematic axis [49]. If $\hat{\mathbf{d}}$ is in the $(x, y)$ plane, a half-quantum vortex can be written

$$
\zeta^{\mathrm{hq}}=\frac{e^{i \varphi / 2}}{\sqrt{2}}\left(\begin{array}{c}
-e^{-i \varphi / 2} \\
0 \\
e^{i \varphi / 2}
\end{array}\right)=\frac{1}{\sqrt{2}}\left(\begin{array}{c}
-1 \\
0 \\
e^{i \varphi}
\end{array}\right) .
$$

In general, the axis about which $\hat{\mathbf{d}}$ winds need not coincide with the vortex core. Figure 3(b) shows a half-quantum vortex where $\hat{\mathbf{d}}$ winds about an axis perpendicular to the vortex line. This vortex is related to that shown in Fig. 3(a) and defined by Eq. (21) by a spin rotation. The resulting spinor wave function may appear quite complicated, but the $\pi$ winding of the nematic axis still allows us to identify the vortex.

Thus circulation is quantized in the polar phase, and indeed one can show that $\pi_{1}\left\{\left[\mathrm{U}(1) \times S^{2}\right] / \mathbb{Z}_{2}\right\}=\mathbb{Z}$. The topological charges $1 / 2,1,3 / 2$, etc., are additive. For example, the state with two half-quantum vortices belongs to the same equivalence class as the singly quantized vortices. This observation shall prove important for understanding the core structure of defects that cross a polar-FM interface.

In addition to singular line defects, the polar phase also supports singular point defects [50,55]: A spherically symmetric point defect, analogous to the 't Hooft-Polyakov monopole in quantum field theory, is formed by choosing $\hat{\mathbf{d}}=\hat{\mathbf{r}}=(\sin \theta \cos \varphi, \sin \theta \sin \varphi, \cos \theta)$ in Eq. (16), such that
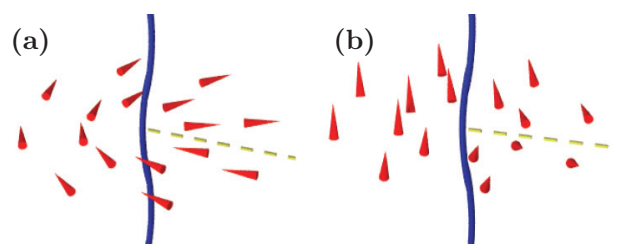

FIG. 3. (Color online) The polar phase of the spin-1 BEC supports half-quantum vortices, constructed as a $\pi$ winding of the condensate phase (which determines the quantized circulation) together with a $\hat{\mathbf{d}} \rightarrow-\hat{\mathbf{d}}$ winding of the nematic axis. The identification $\zeta^{\mathrm{P}}(\phi, \hat{\mathbf{d}})=$ $\zeta^{\mathrm{P}}(\phi+\pi,-\hat{\mathbf{d}})$ keeps the order parameter single valued. (a) Halfquantum vortex as described by Eq. (21). The nematic axis (cones) winds by $\pi$ in the plane perpendicular to the vortex line as the core is encircled. The disclination plane where the $\hat{\mathbf{d}} \leftrightarrow-\hat{\mathbf{d}}$ identification is made is indicated by the dashed line. (b) The winding of the nematic axis need not stay in the plane perpendicular to the vortex line. For example, a half-quantum vortex can also be constructed where $\hat{\mathbf{d}}$ winds in a plane parallel to the vortex line as the defect is encircled. 
the vector field $\hat{\mathbf{d}}$ forms a radial hedgehog structure,

$$
\zeta^{\mathrm{pm}}=\frac{1}{\sqrt{2}}\left(\begin{array}{c}
-e^{-i \varphi} \sin \theta \\
\sqrt{2} \cos \theta \\
e^{i \varphi} \sin \theta
\end{array}\right) .
$$

Here a singular point defect is located at the origin. The two spinor wave function components $\zeta_{ \pm}$form overlapping, singly quantized vortex lines with opposite circulation. The vortex lines are oriented along the $z$ axis, normal to a dark soliton plane (phase kink) in the component $\zeta_{0}$.

\section{TOPOLOGICAL INTERFACE IN A SPIN-1 BEC}

The two phases of the spin-1 BEC exhibit different topological properties of the order parameter, which is manifest in the very different defects they support, as detailed in the previous section. We now consider the behavior of the order parameter and defects when the two phases are realized simultaneously in spatially separated regions within the same ultracold gas, so that an interface between the different ground-state manifolds is formed.

The order-parameter manifolds in the FM and polar phases emerge out of the full symmetry of the condensate wave function as the spin-dependent interaction selects an energetically degenerate subset of all possible wave functions. In particular, the order-parameter space in the FM phase consists of all spinor wave functions that maximize the spin magnitude, $|\langle\hat{\mathbf{F}}\rangle|=1$, everywhere if the texture is uniform. Correspondingly in the polar phase, the order-parameter space is the set of wave functions that have $|\langle\hat{\mathbf{F}}\rangle|=0$ everywhere. These sets are clearly nonoverlapping. However, because they form subsets of the same spin-1 wave function, a continuous connection between spatially separated polar and FM manifolds is possible by exciting the wave function out of its ground-state manifold close to the interface, locally restoring its full symmetry.

In this section we first discuss how the interface may be created in the spinor BEC through local manipulation of the scattering lengths. We then identify the basic interfacecrossing defect states and explicitly construct prototype spinor wave functions to describe them. In Sec. IV we minimize the energy of these spinor wave functions for defect configurations and show how this leads to a rich phenomenology of defect structures.

\section{A. Creating the topological interface}

In order to realize a topological interface in a spinor BEC it is necessary make the system switch continuously between regions of different broken symmetries. Which broken symmetry is preferred in a spin-1 BEC on energetic grounds is determined, as explained in Sec. II A, by the spindependent interaction. Therefore if one can spatially control the interaction strength $c_{2}$, Eq. (5), separate FM and polar regions within the same BEC can be engineered. Specifically, since $c_{2} \propto\left(a_{2}-a_{0}\right)$, this implies changing the ratio $a_{0} / a_{2}$ of the two scattering lengths of colliding spin-1 atoms such that $c_{2}$ changes sign.

The scattering lengths that determine interaction strengths in ultracold-atom systems are routinely manipulated using magnetic Feshbach resonances. However, this technique cannot be used for our present purpose since the strong magnetic fields required would freeze out the spin degree of freedom and destroy the spinor nature of the BEC. The possibility for engineering the scattering lengths in the spinor BEC is instead provided by the use of either optical [88] or microwave-induced Feshbach resonances [89], in which case the fields can be kept sufficiently weak in order not to destroy the spinor nature of the BEC.

We suggest constructing an interface between topologically distinct manifolds in a spinor BEC by local adjustment of the scattering lengths, such that regions with different-sign $c_{2}$ are created [23]. In a spin-1 BEC experiment, the spatial dependence of the scattering lengths can then result in an interface between coexisting FM and polar phases. Doing so, however, presents practical challenges. If an optical Feshbach resonance is used to manipulate one or both scattering lengths, the spatial pattern corresponding to a sharp interface can be imposed using a holographic mask. Optical Feshbach resonances suffer from inelastic losses [88], but these can be kept small for small adjustments of the scattering lengths. Since the spin-dependent interaction is proportional to the difference between $a_{0}$ and $a_{2}$, only a small relative shift is needed to create the interface if $\left|c_{2}\right|$ is small, which is true for both ${ }^{87} \mathrm{Rb}$ and ${ }^{23} \mathrm{Na}$, commonly used in spinor-BEC experiments.

Using a microwave-induced Feshbach resonance avoids the problem of large inelastic losses, but makes engineering the spatial profile more difficult, since, except in specific traps, for example surface microtraps, a microwave field cannot be focused in the same way as the laser. This problem could be overcome by using an optically induced level shift to tune the microwave transition off-resonant. The microwave field could then be applied uniformly across the system, whereas spatial control of the laser field is used to apply the optical tuning only in the region where no shift of $c_{2}$ is required.

\section{B. Construction of prototype interface spinors}

The use of optical or microwave-induced Feshbach resonances can thus realize spatially separated polar and FM phases in the same spin-1 BEC, with the condensate wave function remaining continuous, allowing, in principle, defects to connect across the interface. In order to demonstrate the nontrivial nature of defect penetration across the interface between topologically distinct manifolds, we consider a spin-1 BEC where $c_{2}$ abruptly changes sign at $z=0$. We choose $c_{2}>0$ for $z>0$ and $c_{2}<0$ for $z<0$, such that the interface exists at $z=0$ with the polar phase above it and the FM phase below. In the following, we analytically construct spinor solutions that represent physical wave functions for defects and textures simultaneously in the two different manifolds.

The simplest vortex connection can be identified by considering a singly quantized vortex in both phases, as illustrated schematically in Fig. 4(a). Note that a singly quantized vortex does not mean the same thing in the two phases: the topology that describes vortices is entirely different, one vortex being a product of the broken symmetry manifold $\mathrm{SO}(3)$, with the fundamental homotopy group of two elements, and the other one resulting from the broken symmetry $\left[\mathrm{U}(1) \times S^{2}\right] / \mathbb{Z}_{2}$, with the fundamental homotopy group of integers that represent the 


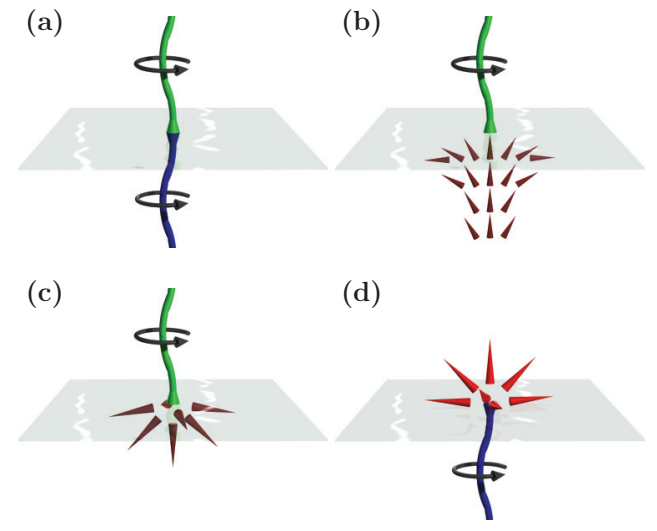

FIG. 4. (Color online) Schematic illustrations of possible vortex connections. The polar phase is above the interface and the FM phase is below. (a) The simplest vortex connection to construct is to consider a singly quantized vortex in either phase. Even though these are different objects, representing different topologies, their wave functions can be joined continuously across the interface. (b) If the singly quantized vortex in the polar phase also contains a $2 \pi$ winding of the nematic axis, its spinor wave function can be made to connect to a spinor representing a coreless vortex in the FM phase. (c) The FM Dirac monopole has a spinor structure similar to the coreless vortex (in fact, the monopole can be continuously unwound into a coreless vortex [56]) and it is therefore possible for it to form the termination point of a singly quantized polar vortex that ends at the interface. (d) Similarly, a polar monopole on the interface can form the termination point of a singly quantized FM spin vortex. The spinor is constructed by noticing that both defects have common phase windings of the spinor components.

number of half quanta of circulation. It is therefore not obvious that these different topological objects can be continuously joined across the interface.

In the following we show how to construct spinor wavefunction solutions that simultaneously represent a singly quantized vortex line in both phases and perforate through the interface with a $2 \pi$ winding of the condensate phase around the vortex line (which we take to be along the $z$ axis). A similar procedure is then extended to other topological defects and textures. The joining of two singly quantized vortex lines can be achieved by changing the sign of either of the spinor components $\zeta_{+}$or $\zeta_{-}$. By appropriate choice of parameters doing so causes the spinor wave function to adjust between the two manifolds by forcing $|\langle\hat{\mathbf{F}}\rangle|$ to switch from 0 to 1 , or else leads to a state which immediately relaxes to the desired configuration. Physically, such a sign change in one of the two spinor components can be obtained by introducing a dark soliton plane (phase kink) in that component at $z=0$. The $\pi$ phase shift across the soliton is then associated with a vanishing density in that spinor component at the soliton core. The BEC wave function, however, remains continuous across the interface, since the remaining spinor components have nonvanishing atom densities also at the position of the soliton plane. The BEC wave function thus connects the two manifolds. In this construction, the switch between polar and FM sides is abrupt. In Sec. IV we will see that as energy is relaxed, the interface acquires a finite width determined by the spin healing length $\xi_{F}$, Eq. (7).
Following this procedure and starting from the expression for a singular vortex in the FM phase, Eq. (10), we can write the spinor wave function connecting two singly quantized vortices across the polar-FM interface explicitly as

$$
\zeta^{1 \leftrightarrow s}=\frac{e^{i \varphi}}{\sqrt{2}}\left(\begin{array}{c}
\sqrt{2} e^{-i \alpha} \cos ^{2} \frac{\beta}{2} \\
\sin \beta \\
\mp \sqrt{2} e^{i \alpha} \sin ^{2} \frac{\beta}{2}
\end{array}\right),
$$

where the negative sign is used on the polar side of the interface and the positive sign on the FM side. Note that only the choice $\beta=\pi / 2$ yields $|\langle\hat{\mathbf{F}}\rangle|=0$ corresponding to an exactly polar state above the interface. However, even for a different $\beta$ the spinor wave function has the appropriate vortex structure and will quickly relax to the polar phase for $z>0$ with a singly quantized vortex. This highlights the general consideration that even though writing exact vortex connections analytically may be very complicated, we have a simple method for finding approximate spinor wave functions representing defect connections.

In Eq. (20) we demonstrated that a singly quantized vortex in the polar phase can include a $2 \pi$ winding of the nematic axis in addition to the $2 \pi$ winding of the condensate phase. Comparing Eq. (20) with the solution for a coreless FM vortex in Eq. (13), we note that these have a similar structure in terms of the complex phases of the spinor components. We can therefore construct an approximate wave function representing the connection of a singly quantized vortex in the polar phase with a coreless vortex on the FM side of the interface by the insertion of a soliton plane in $\zeta_{-}$in Eq. (20). This yields the interface spinor

$$
\zeta^{1 \leftrightarrow \mathrm{cl}}=\frac{1}{\sqrt{2}}\left(\begin{array}{c}
-\sin \beta \\
\sqrt{2} e^{i \varphi} \cos \beta \\
\pm e^{2 i \varphi} \sin \beta
\end{array}\right),
$$

where the positive sign is used on the polar side of the interface and the negative sign on the FM side. Choosing $\beta=\pi / 4$ or $\beta=3 \pi / 4$ yields $|\langle\hat{\mathbf{F}}\rangle|=1$ on the FM side, and specifically the choice $\beta=3 \pi / 4$ approximates the coreless $\operatorname{vortex} \zeta^{\mathrm{cl}}[\mathrm{Eq}$. (13)]. This solution relaxes to the characteristic fountainlike spin profile to yield the state illustrated in Fig. 4(b).

In addition to connecting to another vortex across the interface, a vortex could also terminate on a point defect at the interface. Such solutions can be constructed by joining the monopole spinor wave functions of Eqs. (15) and (22) to vortices with analogous phase windings in each of the spinor components. For example, consider the Dirac monopole in the FM phase [Eq. (15)]. By the same construction that resulted in the interface-crossing defect in Eq. (24) we can connect the singular vortex of Eq. (20) on the polar side to the monopole of Eq. (15) at the interface by inserting a soliton plane into $\zeta_{-}^{\mathrm{D}}$. The resulting spinor wave function,

$$
\zeta^{1 \leftrightarrow \mathrm{D}}=\frac{1}{\sqrt{2}}\left(\begin{array}{c}
\sqrt{2} e^{-2 i \varphi} \cos ^{2} \frac{\theta}{2} \\
e^{-i \varphi} \sin \theta \\
\mp \sqrt{2} \sin ^{2} \frac{\theta}{2}
\end{array}\right),
$$

represents the monopole on the FM side of the interface. Here the negative sign refers to the polar side and the positive sign to 
the FM side. On the polar side the spinor has a structure similar to Eq. (20), thus approximating a singly quantized vortex in the polar phase. The resulting defect configuration is illustrated in Fig. 4(c) and represents a vortex in the polar phase terminating to a monopole at the interface. This defect is closely related to the one shown in Fig. 4(b), as the Dirac monopole and the coreless vortex can be deformed into each other by purely local operations. Note also that in Eq. (25) the Dirac string is represented by the singular polar vortex along the positive $z$ axis and there is no line defect on the FM side. By instead aligning the Dirac string with the negative $z$ axis, the doubly quantized line defect terminates on the monopole from the FM side, while for positive $z$, the spinor still represents a singular vortex connecting to the monopole from the polar side.

In a similar way a point defect (radial hedgehog) in the polar side [Eq. (22)] can be placed on the interface as the termination point of a singular FM vortex. We consider a defect structure with overlapping, singly quantized vortex lines in $\zeta_{ \pm}$, both oriented normal to the interface and of opposite circulation, together with $\pi$ phase kinks in $\zeta_{+}$and $\zeta_{0}$. This spinor wave function can be parametrized as

$$
\zeta^{\mathrm{sv} \leftrightarrow \mathrm{pm}}=\frac{1}{\sqrt{2}}\left(\begin{array}{c}
\mp e^{-i \varphi} \sin \theta \\
\sqrt{2} \cos \theta \\
e^{i \varphi} \sin \theta
\end{array}\right),
$$

using the negative sign on the polar side and the positive sign on the FM side. The resulting structure on the polar side is that of Eq. (22), in which the nematic axis $\hat{\mathbf{d}}$ forms a radial hedgehog $\hat{\mathbf{d}}=\hat{\mathbf{r}}[50,55]$. This represents the polar point defect on the interface. On the FM side the spinor is similar to the singular spin vortex $\zeta^{\mathrm{sv}}$ of Eq. (11) with vortex lines of opposite winding in $\zeta_{ \pm}$. Hence we have constructed on the FM side an approximation to a spin vortex that terminates to the polar monopole at the interface, as illustrated in Fig. 4(d).

Next we show that vortices can also be made to terminate at the interface. In Eq. (24) and in Fig. 4(b), a singular, singly quantized polar vortex perforates the interface to a coreless FM vortex when a $\pi$ phase kink is inserted in $\zeta_{-}$. The resulting defect can be cut in half while still preserving the coherent interface with a continuous order-parameter field by inserting an additional phase kink in $\zeta_{0}$. This allows the vortices on different sides of the interface to move apart:

$$
\begin{aligned}
& \zeta^{\mathrm{cut}}=\frac{1}{\sqrt{2}}\left(\begin{array}{c}
-\sin \beta \\
\sqrt{2} e^{i \varphi} \cos \beta \\
e^{2 i \varphi} \sin \beta
\end{array}\right), \quad \text { for } z>0, \\
& \zeta^{\mathrm{cut}}=\frac{-1}{\sqrt{2}}\left(\begin{array}{c}
\sin \beta \\
\sqrt{2} e^{i \varphi} \cos \beta \\
e^{2 i \varphi} \sin \beta
\end{array}\right), \quad \text { for } z<0,
\end{aligned}
$$

where we may choose $\beta=3 \pi / 4$ as in Eq. (24). One possible configuration is illustrated in Fig. 5(a), where the singular polar vortex and a doubly quantized FM vortex are spatially separated and both terminate on the interface.

Since the vortex lines in the individual spinor components terminate on the soliton planes, it is also possible to consider a state where a vortex exists only on one side of the interface,
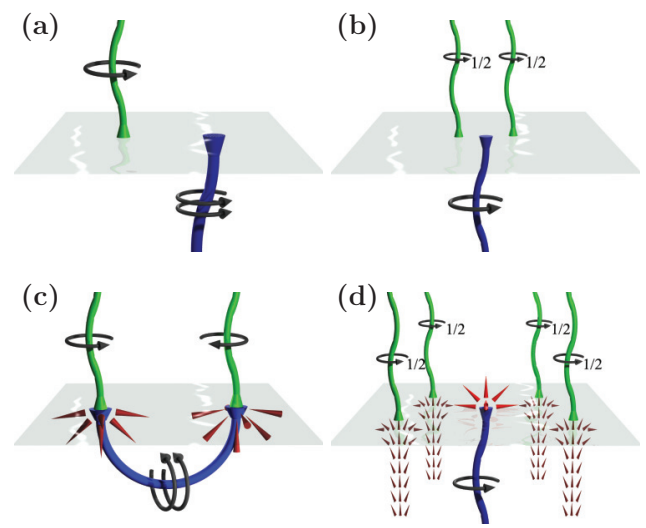

FIG. 5. (Color online) Schematic illustrations of more complicated vortex connections that can be constructed from the basic defect solutions illustrated in Fig. 4. (a) A singly quantized vortex in the polar phase connecting to a doubly quantized vortex on the FM side may be cut in half at the interface and the resulting vortices in the two regions may be moved apart if an additional dark soliton plane is introduced in $\zeta_{0}$. (b) A singly quantized polar vortex can split into two half-quantum vortices when its energy relaxes [63]. A repulsive force between the half-quantum vortices makes the splitting energetically favorable. The splitting mechanism can then yield a state where a FM vortex connects to a pair of half-quantum vortices. (c) A Dirac dipole can be constructed by joining the Dirac strings of a Dirac monopole and an antimonopole [56]. Placed on the interface, the dipole connects to two singly quantized vortices on the polar side. (d) Rotation may cause interface-crossing vortex structures to nucleate. Here four nucleated vortex complexes are drawn together with a singular vortex already present.

for instance,

$$
\begin{gathered}
\zeta^{\mathrm{pv}}=\frac{1}{\sqrt{2}}\left(\begin{array}{c}
-\sin \beta \\
\sqrt{2} e^{i \varphi} \cos \beta \\
e^{2 i \varphi} \sin \beta
\end{array}\right), \quad \text { for } z>0, \\
\zeta^{\mathrm{pv}}=-\frac{1}{\sqrt{2}}\left(\begin{array}{c}
\sin \beta \\
\sqrt{2} \cos \beta \\
\sin \beta
\end{array}\right), \quad \text { for } z<0 .
\end{gathered}
$$

In addition to singly quantized vortices also half-quantum vortices are possible in the polar phase [Eq. (21)]. A singly quantized vortex can split into two half-quantum vortices while preserving the topology, and such splitting can be energetically favorable [63]. The defect configuration of perforating singly quantized vortices in Fig. 4(a) can therefore also deform to a state in which a singly quantized FM vortex continuously connects to a pair of half-quantum vortices as illustrated in Fig. 5(b). We will demonstrate in the next section that this state does indeed appear as a consequence of energy minimization.

The vortices and monopoles in the spin-1 BEC are made up of vortex lines and soliton planes in the individual spinor components. The construction of the interface-crossing defect solutions was achieved by identifying defects in the two phases that have a similar combination of vortex lines, and using soliton planes to achieve the switch between polar and FM manifolds. Dark solitons have been phase imprinted experimentally $[90,91]$. Phase imprinting of vortex lines in a BEC by transferring angular momentum from an electromagnetic field 
has been proposed theoretically [92-96] and several of the techniques have also been realized in experiments [97-100]. In spinor BECs, coreless vortices and related textures have been prepared by adiabatic ramping of a magnetic field along the trap axis [70,72,73], or by population transfer using Laguerre-Gaussian laser [71]. More complicated vortices and textures could be imprinted using proposed techniques for creating vortex rings [45,101].

Other interface defect configurations can be constructed by combining more elementary defect connections. For instance, in the FM phase the Dirac monopole can be turned inside out to form an antimonopole, in which the spins point radially inward. By joining the Dirac strings of a Dirac monopole and an antimonopole, a Dirac dipole can be constructed [56]. In Fig. 5(c), we illustrate a state where such a dipole is placed on the polar-FM interface. The Dirac string forms a doubly quantized vortex line in the FM phase, connecting the two monopoles. Consequently, the Dirac dipole can form the termination points of two oppositely winding singly quantized vortices [see the construction that led to Eq. (25)].

\section{CORE STRUCTURE OF INTERFACE-CROSSING DEFECTS}

\section{A. Core deformation of interface-crossing defect solutions}

In the preceding section we constructed the prototype spinor wave functions for the interface-crossing defect solutions connecting defects in the FM and polar phases. We showed that such solutions can be formed by combinations of elementary vortex lines and dark soliton (phase kink) planes that could be experimentally prepared by phase imprinting. Here we use the constructed spinor wave functions for the defect configurations as initial states for numerical studies of the defect stability. Provided that prototype spinors sufficiently closely approximate the local energetic minimum configuration, the initial states quickly relax to the targeted defect structure.

By numerical simulations we can determine the energetically preferred core structures and the energetic stability of the defects. In order to do so we minimize the free energy in the rotating frame [Eq. (4)] by propagating the coupled Gross-Pitaevskii equations, derived from Eq. (2), in imaginary time using a split-step algorithm [102]. We assume the slightly elongated trap, defined by Eq. (3). The initial state prototype spinor wave functions are given in Sec. IIIB. We choose the spin-independent nonlinearity $c_{0}=2.0 \times 10^{4} \hbar \omega l^{3}$, where $l=(\hbar / m \omega)^{1 / 2}$ is the transverse oscillator length. For ${ }^{87} \mathrm{Rb}$ in a trap with $\omega=2 \pi \times 10 \mathrm{~Hz}$ these parameters correspond to $10^{6}$ atoms. The spin-dependent nonlinearity $c_{2}$ is allowed to vary.

In Ref. [63] it was demonstrated that the core of a singly quantized vortex in the FM phase of spin-1 BEC deforms by locally rotating the spin vector so that the vortex lines in the individual spinor components in the appropriate basis representation move apart. The singular vortex line then no longer represents a vanishing atom density, but is occupied by atoms with zero spin magnitude as in the polar phase of the spin-1 BEC. The FM vortex line singularity filled by atoms in the polar phase becomes energetically favorable by allowing a larger core size and a correspondingly smaller bending energy.
The core deformation can be understood from the energetics associated with the hierarchy of characteristic length scales determined by the interaction strengths: the size of the filled core is determined by the spin healing length $\xi_{F}$, Eq. (7), which is usually larger than the density healing length $\xi_{n}$, Eq. (6), that sets the size of a core with vanishing density.

Similarly, a singly quantized vortex in the polar phase was shown to lower its energy by spontaneously breaking axial symmetry, splitting into a pair of singular half-quantum vortices [63]. This again avoids depleting the atom density in the vortex core: at the location of the singularities $|\langle\hat{\mathbf{F}}\rangle|=1$, with spins antialigning in the two cores. The two vortices form an extended core region where the order parameter is excited out of the polar ground-state manifold. The size of the core region is then enlarged to be on the order of $\xi_{F}$, with a corresponding decrease in bending energy. The overall topology is preserved away from the two singularities. Inside the extended core region, the splitting of the singly quantized vortex locally deforms the nematic $\hat{\mathbf{d}}$ field that describes the order parameter [see Eq. (17)], and a disclination plane, where the identification $\hat{\mathbf{d}} \leftrightarrow-\hat{\mathbf{d}}$ is made, appears between the vortex lines. Thus on a loop encircling only one line singularity, both condensate phase and nematic axis wind by $\pi$. The splitting of the singly quantized vortex is closely related to the deformation of a point defect into a half-quantum vortex ring [55]. In the $2 \mathrm{D}$ cross section of the ring, the diametrically opposite points on the ring correspond to half-quantum vortices with antialigned spins in the cores. In Ref. [60] dynamic nucleation of half-quantum vortices under energy dissipation was studied, demonstrating formation of a square vortex lattice. To get a simple qualitative picture of the interactions between half-quantum vortices one may consider the corresponding problem in a two-component BEC. In a nonrotating uniform system it was argued that the repulsive force between vortices with opposite core polarizations falls off as $1 / R^{3}$ [103].

It was demonstrated in Eq. (23) how singly quantized vortices in the FM and polar phases can be connected across the polar-FM interface, despite the fact that these are two topologically different defects. As the energy is minimized, this interface-crossing defect deforms by a mechanism analogous to that described above for the singly quantized vortices in the purely FM and polar BECs. The resulting structure is shown in Fig. 6. On the polar side of the interface, the splitting of the singly quantized vortex into two half-quantum vortices is recognized from the deformation of the nematic field, which shows the characteristic $\pi$ winding around each singularity, and the formation of the disclination plane. The order parameter is excited out of the $|\langle\hat{\mathbf{F}}\rangle|=0$ ground-state manifold, to reach $|\langle\hat{\mathbf{F}}\rangle|=1$ at the singular lines. The pair of half-quantum vortices connects across the interface to the singly quantized FM vortex. This, in turn, exhibits the local rotation of the spin vector, allowing the core region to fill by mixing FM and polar phases, with $|\langle\hat{\mathbf{F}}\rangle|=0$ on the singularity.

The relaxed interface-crossing vortex structure is thus recognized as that illustrated schematically in Fig. 5(b), and the deformation is understood in terms of the characteristic length scales set by the atom-atom interactions. In the purely polar or FM condensate, the core-deformed, singly quantized vortices are energetically stable [63]. For the parameter values 


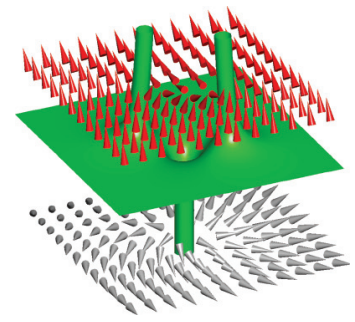

FIG. 6. (Color online) Deformation of connecting singly quantized vortices. The green (gray) $|\langle\hat{\mathbf{F}}\rangle|$ isosurface shows the interface and the cores of singular defects. The polar phase is above the interface. Energy relaxation causes the singly quantized vortex on the polar side to split into a pair of half-quantum vortices with $|\langle\hat{\mathbf{F}}\rangle|>0$ cores on the polar side, recognizable by the characteristic $\pi$ winding of $\hat{\mathbf{d}}$ [red (dark gray) cones]. These connect across the interface to a singly quantized FM vortex [cf. Fig. 5(b)]. A local rotation of the spin vector (light gray cones) allows the core region to fill with atoms with $|\langle\hat{\mathbf{F}}\rangle|<1$. $\left(\left|c_{2}\right|=1.0 \times 10^{4} \hbar \omega l^{3}\right.$ and $\Omega=0.20 \omega$. $)$

investigated, the configuration in Fig. 6 ultimately decays for very long relaxation times in our simulations.

We do, however, find an energetically stable deformation of a singly quantized polar vortex connecting across the interface if instead of starting from $\zeta^{1 \leftrightarrow s}$ of Eq. (23), we minimize the energy of $\zeta^{1 \leftrightarrow \mathrm{cl}}$ from Eq. (24). This spinor describes a singly quantized polar vortex connecting across the interface to a coreless vortex. [A topologically equivalent configuration can be constructed by allowing the polar vortex to terminate on a Dirac monopole, Eq. (25).] Minimizing the energy leads
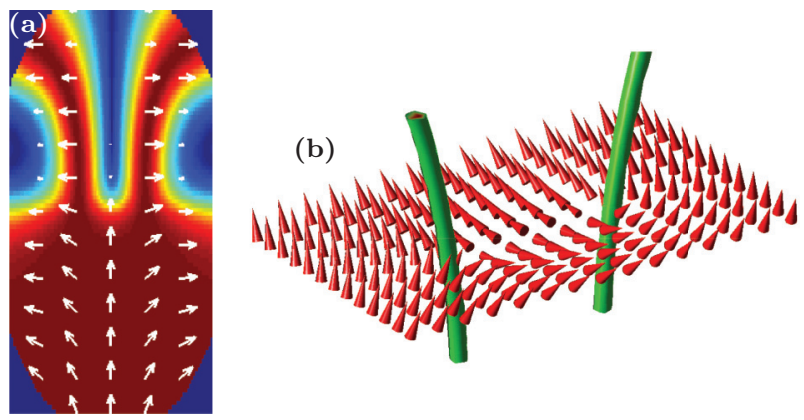

FIG. 7. (Color online) Minimizing the energy of Eq. (24), corresponding to Fig. 4(b) results in a splitting of the singly quantized polar vortex, while the characteristic fountainlike spin structure of the coreless vortex is established in the FM part of the cloud. (a) The magnitude of the spin $[|\langle\hat{\mathbf{F}}\rangle|=1$ is dark red (dark gray) with long arrows] shows the interface after relaxation of the energy, and the filled cores, with $|\langle\hat{\mathbf{F}}\rangle|=1$ at the singularity, of two half-quantum vortices in the polar part. White arrows show the spin vector and indicate the coreless vortex in the FM part, and how the spin structure connects to the vortex cores across the interface. This result was obtained using $\left|c_{2}\right|=2.5 \times 10^{2} \hbar \omega l^{3}$ and $\Omega=0.12 \omega$. (b) The half-quantum vortices may be identified by the winding of the nematic axis $\hat{\mathbf{d}}$ (unoriented but shown as cones to emphasize winding). This displays the characteristic $\hat{\mathbf{d}} \rightarrow-\hat{\mathbf{d}}$ winding as any single vortex core is encircled. The two cores are joined by a disclination plane. Note that away from the core region the original topology of the singly quantized vortex is preserved. Here a stronger spin-dependent nonlinearity has been used to get more sharply defined FM cores: $\left|c_{2}\right|=1.0 \times 10^{4} \hbar \omega l^{3}, \Omega=0.19 \omega$.
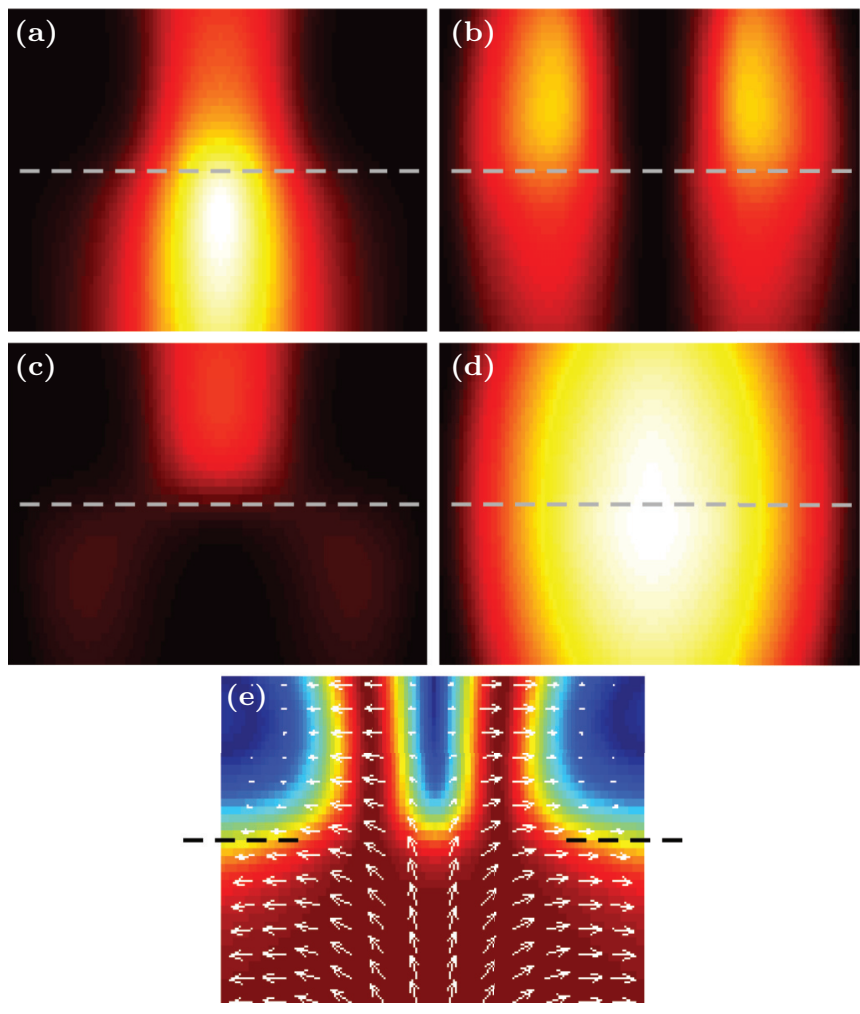

FIG. 8. (Color online) The condensate wave function varies smoothly across the polar-FM interface, showing that the defect states cross the interface smoothly, connecting the two topologies. (a)-(c) Densities in the individual spinor components $\zeta_{+}, \zeta_{0}$ and $\zeta_{-}$, respectively. These vary smoothly and do not vanish simultaneously on the interface. The position of the interface, defined as the plane where $c_{2}$ changes sign, is indicated by the dashed line. (d) The smooth variation of the nonzero atomic density across the interface shows the continuity of the condensate wave function. (e) $\langle\hat{\mathbf{F}}\rangle$ shown as color map [dark red (dark gray) with long arrows at $|\langle\hat{\mathbf{F}}\rangle|=1$ ] and vector field (white arrows). Note how the spin structure connects smoothly across the interface, in particular connecting the FM cores of the half-quantum vortices continuously to the fountain structure of the spin in the coreless vortex. The magnitude of the spin also shows how the interface has acquired a finite width.

to the deformation shown in Fig. 7. On the FM side of the interface, the spin structure acquires the fountainlike structure characteristic of the coreless vortex, as shown by the white arrows in Fig. 7(a). Here the frequency of rotation determines the direction of the spin vector at the edge of the cloud as the angular momentum in the FM phase adapts to the imposed rotation.

On the polar side of the interface we recognize the splitting of the singly quantized vortex into a pair of half-quantum vortices, identified by the $\pi$ winding of the nematic axis $\hat{\mathbf{d}}$ around each vortex [Fig. 7(b)], preserving the overall topology. As before, in the core region the order parameter is excited out of the ground-state manifold, with $|\langle\hat{\mathbf{F}}\rangle|=1$ on the singular lines. Figure 7(a) shows how the spin texture connects smoothly across the interface.

The continuity of the relaxed spinor wave function is further demonstrated in Fig. 8, giving a detailed picture of the interface region. In the relaxed state, the total atom density remains 
(a)

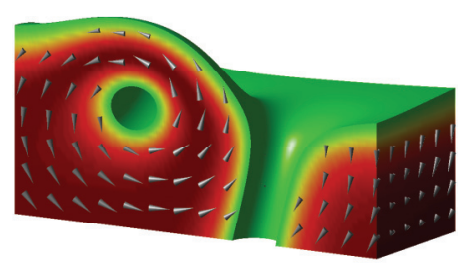

(b)

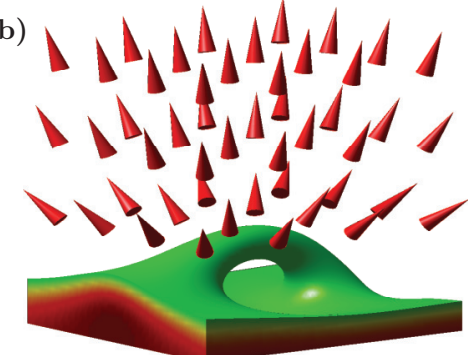

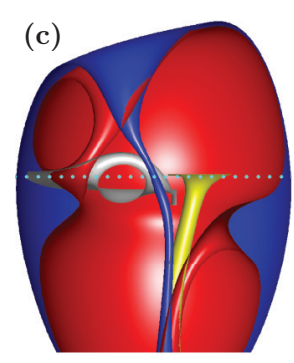

FIG. 9. (Color online) In Fig. 4(d) a polar point defect forms the termination of a FM spin vortex. As the energy relaxes, the point defect deforms into an archlike, singular defect line that connects to the interface at both ends. (a) An isosurface of the spin magnitude is shown in green (light gray). The spin magnitude rises to 1 (dark red, black) on the FM side of the interface $(z<0)$ and inside the line defect. Gray cones indicate the spin vector. (b) Away from the defect, the $\hat{\mathbf{d}}$ vector field (cones) in the polar phase retains the hedgehog structure of the original monopole. (c) In the trial wave function, Eq. (26), both the FM spin vortex and the polar monopole are formed by exactly overlapping vortex lines of opposite winding in $\zeta_{+}^{\mathrm{sv} \leftrightarrow \mathrm{pm}}$ and $\zeta_{-}^{\mathrm{sv} \leftrightarrow \mathrm{pm}}$. Constant-density surfaces for $n\left|\zeta_{+}\right|^{2}$ (red, medium gray) and $n\left|\zeta_{-}\right|^{2}$ (blue, dark gray) show how the arch-shaped line defect is formed by deformation of these vortex lines, such that they no longer overlap close to the interface. The half-quantum vortex line (above the interface) and the spin vortex (below the interface) are indicated by silver and gold (light gray) spin isosurfaces at $|\langle\hat{\mathbf{F}}\rangle|=0.9$ and $|\langle\hat{\mathbf{F}}\rangle|=0.5$, respectively. $\left(\left|c_{2}\right|=5.0 \times 10^{2} \hbar \omega l^{3}\right.$ and $\Omega=0$.)

nonvanishing at the interface and varies smoothly across it. The populations of the individual spinor components are also continuous across the interface. The continuity of the defect-carrying spinor wave function as it crosses between the different broken symmetries means that it represents a continuous connection of defects across the interface. In Fig. 8, the position where $c_{2}$ changes sign is indicated by a dashed line, and $|\langle\hat{\mathbf{F}}\rangle|$ shown in panel (e) shows the finite width of the interface region after energy relaxation.

The vortex core structures are particularly intriguing and complex when a vortex terminates to a point defect on the interface. We study a singular FM vortex terminating to a radial hedgehog at the interface ( $\hat{\mathbf{d}}$ forms a hemispherical hedgehog on the polar side), as depicted schematically in Fig. 4(d). Unlike the FM Dirac monopole, the polar monopole cannot unwind into simpler vortex configuration. Therefore, while the connection depicted in Fig. 4(b) is equivalent to Fig. 4(c) and leads to the same energy-minimizing defect configuration, Fig. 4(d) is topologically distinct from all other vortex connections.

A constructed prototype spinor wave function representing a singular FM vortex terminating to a hedgehog point defect is given by Eq. (26). At the point defect singularity the atom density is zero. The density depletion at the defect core is energetically costly, and if $\xi_{F}$, Eq. (7), is sufficiently large in comparison with $\xi_{n}$, Eq. (6), the energy cost can be reduced by deforming the point defect into a semicircular line defect whose ends attach to the interface. The resulting archlike defect is shown in Fig. 9 together with the spin structure in the FM core (a) and the nematic axis away from the defect on the polar side of the interface (b). The deformation of the defect, schematically illustrated in Fig. 10, is local and the topological charge of the monopole is retained: away from the defect, the radial hedgehog structure of $\hat{\mathbf{d}}$ is preserved. This implies that on any closed loop through the arch, $\hat{\mathbf{d}}$ must turn by $\pi$. Consequently, single valuedness of the spinor wave function requires the condensate phase to also turn by $\pi$, and we infer that the arch-shaped line defect is a half-quantum vortex. This Alice arch resembles the upper hemispheric part of the Alice ring — a closed half-quantum vortex ring that exhibits the topological charge of a point defect over any surface enclosing the defect [55]. Alice rings also appear in high-energy physics [104] with a topological charge similar to the magnetic "Cheshire" charge [105].

The deformation of the point defect at the interface to an Alice arch results from a complex interplay between the energetic considerations, topology, and the length scale hierarchy in the system. The two characteristic healing lengths determine whether the energy cost of forming the extended vortex core region, where the singularity is occupied by the atoms in the FM phase, offsets the energy cost of the density depletion at a point defect (with a vanishing density at the singularity). We find an abrupt transition point to an Alice arch when $c_{2} \lesssim 0.5 c_{0}$. For larger values of $c_{2}$ the point defect at the interface is preferred to the arch defect. The sharp threshold for the deformation can be understood by the topology. For the point defect there cannot be a singular point with $|\langle\mathbf{F}\rangle|=1$ and a spherically symmetric core region with $|\langle\hat{\mathbf{F}}\rangle|>0$. The nematic axis forms a radial hedgehog structure and this would then imply that the spin vector (always orthogonal to $\hat{\mathbf{d}}$ ) would have to form a continuous tangent vector field for the
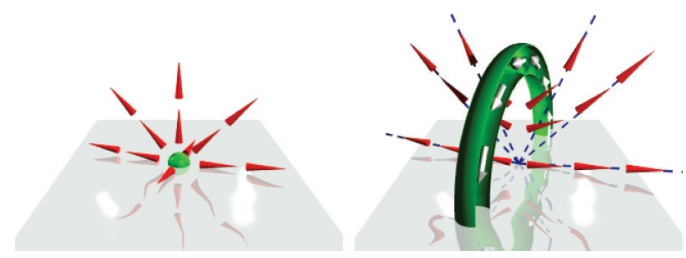

FIG. 10. (Color online) Deformation of the hedgehog point defect (left) on the interface into an arch-shaped line defect (right). Red (gray) cones represent the (unoriented) $\mathbf{d}$ field; the defect cores are shown as a green (gray) sphere and torus, respectively. The deformation creates a hole in the point-defect core, allowing it to expand into the line defect by locally deforming the $\hat{\mathbf{d}}$ field. Away from the line defect, the asymptotic hedgehog structure is preserved. (Dashed lines indicate unperturbed $\hat{\mathbf{d}}$ field for reference.) On any closed loop through the arch, $\hat{\mathbf{d}}$ therefore winds to $-\hat{\mathbf{d}}$, identifying the defect as a half-quantum vortex line. The line-defect core is filled with atoms with $|\langle\hat{\mathbf{F}}\rangle|>0$; spin vector indicated by silver arrows. 
spherically symmetric object which is not possible ("hairy ball" theorem). Therefore the point defect has to deform to a ring or an arch-shaped defect at the interface before the transition from the zero-density singularity to the $|\langle\hat{\mathbf{F}}\rangle|=1$ singularity with nonvanishing density is possible. The sharp deformation threshold also appears in the stability analysis of a singular point defect and the Alice ring [55].

We note that the arch defect is unstable towards drifting out of the cloud due to the density gradient resulting from the harmonic trapping potential. It could potentially be stabilized using a weak pinning laser to create a small density depletion at the center of the trap.

The structure of the arch defect emerging from the point defect may be understood by studying the individual spinor wave function components. In the point defect with vanishing density at the singularity, the overlapping vortex lines in $\zeta_{ \pm}$ intersect with the soliton plane in $\zeta_{0}$. In the prototype spinor wave function $\zeta^{\mathrm{sv} \leftrightarrow \mathrm{pm}}$ an additional soliton plane is present in $\zeta_{+}$to account for the switch from the polar to the FM side of the interface. In Ref. [55] the deformation of the spherically symmetric point defect was explained by a local separation of the vortex lines in $\zeta_{ \pm}$such that they no longer overlap at the soliton plane. Here the additional soliton plane in $\zeta_{+}$cuts the vortex line in $\zeta_{+}$at the interface, separating the two parts. The spinor components $\zeta_{ \pm}$are shown in Fig. 9(c). The positions of the Alice arch and the FM vortex are indicated, and we find how in the polar part of the cloud, the separated vortex lines make up the semicircular half-quantum vortex. On the FM side, the unbroken vortex line in $\zeta_{-}$and the $z<0$ part of the vortex line in $\zeta_{+}$form the spinor wave function of the FM vortex whose core is filled by the vortex-free $\zeta_{0}$ component.

At high rotation frequencies of the trap, we find nucleation of interface-crossing defects in the energy minimization. Provided that the appropriate instability for nucleating vortices is triggered, the emergence of defect configurations where a halfquantum vortex connects to a coreless vortex spontaneously emerges, as they lower the energy of the system in a sufficiently rapidly rotating trap. An example is shown in Fig. 11, in which four interface-crossing vortices nucleate. On the polar side of the interface, four singular lines appear, on which $|\langle\hat{\mathbf{F}}\rangle|=1$.
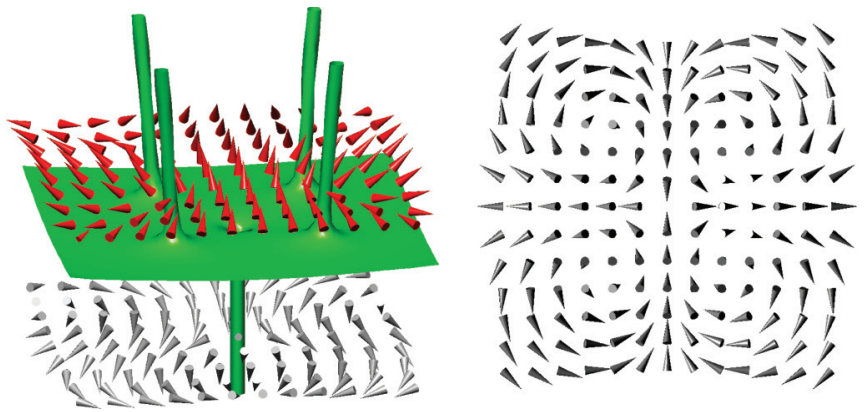

FIG. 11. (Color online) Left: Isosurface of $|\langle\hat{\mathbf{F}}\rangle|$ showing the cores of singular defects, together with $\hat{\mathbf{d}}$ (red or dark gray cones) on the polar side of the interface, and $\langle\hat{\mathbf{F}}\rangle$ (light gray cones) in the FM part. Four nucleated vortices are identified as polar half-quantum vortices connecting across the interface to nonsingular coreless vortices. Right: Top-down view of $\langle\hat{\mathbf{F}}\rangle$ in the FM part, showing the four coreless vortices.
These vortices may be identified as half-quantum vortices through the winding of the nematic axis. On the FM side, the order parameter remains nonsingular. However, the spin texture reveals that each of the four half-quantum vortices connects to a coreless vortex.

\section{CONCLUSIONS}

Interfaces between topologically distinct ground-state manifolds play an important role in several areas of physics, many of which are difficult or impossible to access experimentally. Here we have given a detailed analysis of our proposal for how topological interface physics of defects and textures can be studied in ultracold atomic gases [23]. We considered the specific example of a spin-1 BEC with spatially separated polar and FM regions within a continuous condensate wave function. For a number of representative, interface-crossing defect states, we provided detailed constructions of prototype spinor wave functions by considering how basic vortex and monopole solutions with similar spinor structure represent different topological objects in the two phases. The resulting wave functions are built from vortex lines and soliton planes in the individual spinor components that could be phase imprinted using existing experimental techniques [45,90,91,97-101].

The energetic stability and energy-minimizing core structures of the interface-crossing defect configurations were analyzed by numerically minimizing the energy of the prototype wave functions. The resulting spinor states demonstrate how the condensate wave function smoothly interpolates between the two ground-state manifolds by locally restoring its full symmetry, thereby establishing a coherent interface through which defects may connect continuously. In particular, we demonstrated the energetically stable connection of a coreless vortex to a pair of half-quantum vortices, and the formation of an Alice arch: the deformation of a point defect at the interface into an arch-shaped half-quantum vortex line that preserves the topological charge.

In order to demonstrate the basic principle of the topological interface physics in ultracold atoms, we have concentrated in this work on a relatively simple and accessible example of defect perforation across constructed interfaces in spin-1 BECs. The interface analysis, however, can also be applied to more complex systems, such as spin-2 [27,28,64] and spin-3 [29,30] BECs, where, for example, non-Abelian defects are predicted $[65,66]$. Other particularly promising platforms for topological interface studies are strongly correlated atoms in optical lattices [106-108] exhibiting also quantum phase transitions and potential analogs of exotic superconductivity [10] in crystal lattices.

Moreover, the interface scheme may be used to investigate nonequilibrium dynamical scenarios for production of topological defects and textures in phase transitions. An intriguing possibility is production of topological defects in experiments inspired by brane-inflation models, where brane annihilation leads to formation of defects [8,9]. In a FM $\mathrm{BEC}$, a region of polar phase can be created by locally shifting the spin-dependent interaction strength. The resulting phase boundaries then form two-dimensional analogues of $D$-branes. When the interaction shift is removed, the polar region collapses, simulating brane-antibrane annihilation, and 
resulting defects can be observed in the cloud. This is similar to a recent experiment in ${ }^{3} \mathrm{He}$ [14], where, however the defects are more difficult to observe directly.

Defect production may also result from dynamic instabilities, for example arising from superfluid counterflow between the FM and polar phases. The boundary between two fluids moving with respect to each other becomes unstable if the relative velocity exceeds some critical value, leading to excitations on the interface. This phenomenon is well understood in classical fluid mechanics and is known as the Kelvin-Helmholtz instability [109]. An analogous superfluid Kelvin-Helmholtz instability has been shown to occur at the interface between superfluid ${ }^{3} \mathrm{He} A$ and $B$, providing another active area of research related to interfaces between different ordered phases [13]. Vortices in the $A$ phase cause counterflow against the initially vortex-free $B$ phase. As the relative velocity exceeds a critical value, vortices nucleate from the interface into the $B$ phase. A superfluid Kelvin-Helmholtz instability has also been predicted in phase-separated twocomponent BECs [110,111].

\section{ACKNOWLEDGMENTS}

We thank D. J. Papoular for enlightening discussions. Financial support from the Leverhulme trust is gratefully acknowledged.
[1] N. D. Mermin, Rev. Mod. Phys. 51, 591 (1979).

[2] D. Vollhardt and P. Wölfle, The Superfluid Phases of Helium 3 (Taylor \& Francis Ltd, London, 1990).

[3] J. Stenger, S. Inouye, D. M. Stamper-Kurn, H. Miesner, A. P. Chikkatur, and W. Ketterle, Nature (London) 396, 345 (1998).

[4] Y. Kawaguchi and M. Ueda, Phys. Rep. 520, 253 (2012).

[5] D. M. Stamper-Kurn and M. Ueda, arXiv:1205.1888.

[6] T. W. B. Kibble, J. Phys. A 9, 1387 (1976).

[7] A. Vilenkin and E. P. S. Shellard, Cosmic Strings and Other Topological Defects (Cambridge University Press, Cambridge, England, 1994).

[8] G. Dvali and S.-H. H. Tye, Phys. Lett. B 450, 72 (1999).

[9] S. Sarangi and S.-H. H. Tye, Phys. Lett. B 536, 185 (2002).

[10] J. A. Bert, B. Kalisky, C. Bell, M. Kim, Y. Hikita, H. Y. Hwang, and K. A. Moler, Nat. Phys. 7, 767 (2011).

[11] M. M. Salomaa, Nature (London) 326, 367 (1987).

[12] G. E. Volovik, The Universe in a Helium Droplet (Oxford University Press, New York, 2003).

[13] A. P. Finne, V. B. Eltsov, R. Hänninen, N. B. Kopnin, J. Kopu, M. Krusius, M. Tsubota, and G. E. Volovik, Rep. Prog. Phys. 69, 3157 (2006).

[14] D. I. Bradley, S. N. Fisher, A. M. Guenault, R. P. Haley, J. Kopu, H. Martin, G. R. Pickett, J. E. Roberts, and V. Tsepelin, Nat. Phys. 4, 46 (2008).

[15] W. H. Zurek, Nature (London) 317, 505 (1985).

[16] C. Bäuerle, Y. M. Bunkov, S. N. Fisher, H. Godfrin, and G. R. Pickett, Nature (London) 382, 332 (1996).

[17] V. M. H. Ruutu, V. B. Eltsov, A. J. Gill, T. W. B. Kibble, M. Krusius, Y. G. Makhlin, B. Plaçais, G. E. Volovik, and W. Xu, Nature (London) 382, 334 (1996).

[18] C. N. Weiler, T. W. Neely, D. R. Scherer, A. S. Bradley, M. J. Davis, and B. P. Anderson, Nature (London) 455, 948 (2008).

[19] M. Nitta, K. Kasamatsu, M. Tsubota, and H. Takeuchi, Phys. Rev. A 85, 053639 (2012).

[20] R. A. Battye, N. R. Cooper, and P. M. Sutcliffe, Phys. Rev. Lett. 88, 080401 (2002).

[21] C. M. Savage and J. Ruostekoski, Phys. Rev. Lett. 91, 010403 (2003).

[22] J. Ruostekoski, Phys. Rev. A 70, 041601 (2004).

[23] M. O. Borgh and J. Ruostekoski, Phys. Rev. Lett. 109, 015302 (2012).

[24] T.-L. Ho, Phys. Rev. Lett. 81, 742 (1998).

[25] T. Ohmi and K. Machida, J. Phys. Soc. Jpn. 67, 1822 (1998).

[26] F. Zhou, Int. J. Mod. Phys. B 17, 2643 (2003).
[27] M. Koashi and M. Ueda, Phys. Rev. Lett. 84, 1066 (2000).

[28] C. V. Ciobanu, S.-K. Yip, and T.-L. Ho, Phys. Rev. A 61, 033607 (2000).

[29] R. Barnett, A. Turner, and E. Demler, Phys. Rev. Lett. 97, 180412 (2006).

[30] L. Santos and T. Pfau, Phys. Rev. Lett. 96, 190404 (2006).

[31] M. M. Salomaa and G. E. Volovik, Rev. Mod. Phys. 59, 533 (1987).

[32] N. Manton and P. Sutcliffe, Topological Solitons (Cambridge University Press, Cambridge, England, 2004).

[33] E. B. Bogomolny, Sov. J. Nucl. Phys. 24, 449 (1976).

[34] R. Jackiw and C. Rebbi, Phys. Rev. D 13, 3398 (1976).

[35] L. Faddeev and A. J. Niemi, Nature (London) 387, 58 (1997).

[36] T. Busch and J. R. Anglin, Phys. Rev. Lett. 87, 010401 (2001).

[37] P. Öhberg and L. Santos, Phys. Rev. Lett. 86, 2918 (2001).

[38] P. Kevrekidis, H. Nistazakis, D. Frantzeskakis, B. Malomed, and R. Carretero-González, Eur. Phys. J. D 28, 181 (2004).

[39] N. G. Berloff, Phys. Rev. Lett. 94, 120401 (2005).

[40] U. Shrestha, J. Javanainen, and J. Ruostekoski, Phys. Rev. Lett. 103, 190401 (2009).

[41] C. Yin, N. G. Berloff, V. M. Pérez-García, D. Novoa, A. V. Carpentier, and H. Michinel, Phys. Rev. A 83, 051605 (2011).

[42] H. E. Nistazakis, D. J. Frantzeskakis, P. G. Kevrekidis, B. A. Malomed, and R. Carretero-González, Phys. Rev. A 77, 033612 (2008).

[43] R. Carretero-González, D. J. Frantzeskakis, and P. G. Kevrekidis, Nonlinearity 21, R139 (2008).

[44] K. Kasamatsu, M. Tsubota, and M. Ueda, Phys. Rev. Lett. 91, 150406 (2003).

[45] J. Ruostekoski and J. R. Anglin, Phys. Rev. Lett. 86, 3934 (2001).

[46] U. Al Khawaja and H. Stoof, Nature (London) 411, 918 (2001).

[47] T. Kawakami, T. Mizushima, M. Nitta, and K. Machida, Phys. Rev. Lett. 109, 015301 (2012).

[48] S.-K. Yip, Phys. Rev. Lett. 83, 4677 (1999).

[49] U. Leonhardt and G. Volovik, JETP Lett. 72, 46 (2000).

[50] H. T. C. Stoof, E. Vliegen, and U. Al Khawaja, Phys. Rev. Lett. 87, 120407 (2001).

[51] T. Isoshima and K. Machida, Phys. Rev. A 66, 023602 (2002).

[52] T. Mizushima, K. Machida, and T. Kita, Phys. Rev. A 66, 053610 (2002).

[53] T. Mizushima, K. Machida, and T. Kita, Phys. Rev. Lett. 89, 030401 (2002). 
[54] J.-P. Martikainen, A. Collin, and K.-A. Suominen, Phys. Rev. A 66, 053604 (2002).

[55] J. Ruostekoski and J. R. Anglin, Phys. Rev. Lett. 91, 190402 (2003).

[56] C. M. Savage and J. Ruostekoski, Phys. Rev. A 68, 043604 (2003).

[57] J. W. Reijnders, F. J. M. van Lankvelt, K. Schoutens, and N. Read, Phys. Rev. A 69, 023612 (2004).

[58] E. J. Mueller, Phys. Rev. A 69, 033606 (2004).

[59] H. Saito, Y. Kawaguchi, and M. Ueda, Phys. Rev. Lett. 96, 065302 (2006).

[60] A.-C. Ji, W. M. Liu, J. L. Song, and F. Zhou, Phys. Rev. Lett. 101, 010402 (2008).

[61] M. Takahashi, V. Pietilä, M. Möttönen, T. Mizushima, and K. Machida, Phys. Rev. A 79, 023618 (2009).

[62] T. P. Simula, J. A. M. Huhtamäki, M. Takahashi, T. Mizushima, and K. Machida, J. Phys. Soc. Jpn. 80, 013001 (2011).

[63] J. Lovegrove, M. O. Borgh, and J. Ruostekoski, Phys. Rev. A 86, 013613 (2012).

[64] G. W. Semenoff and F. Zhou, Phys. Rev. Lett. 98, 100401 (2007).

[65] J. A. M. Huhtamäki, T. P. Simula, M. Kobayashi, and K. Machida, Phys. Rev. A 80, 051601 (2009).

[66] M. Kobayashi, Y. Kawaguchi, M. Nitta, and M. Ueda, Phys. Rev. Lett. 103, 115301 (2009).

[67] R. Barnett, A. Turner, and E. Demler, Phys. Rev. A 76, 013605 (2007).

[68] H. Takeuchi and M. Tsubota, J. Phys. Soc. Jpn. 75, 063601 (2006).

[69] K. Kasamatsu, H. Takeuchi, M. Nitta, and M. Tsubota, J. High Energy Phys. 11 (2010) 068.

[70] A. E. Leanhardt, Y. Shin, D. Kielpinski, D. E. Pritchard, and W. Ketterle, Phys. Rev. Lett. 90, 140403 (2003).

[71] L. S. Leslie, A. Hansen, K. C. Wright, B. M. Deutsch, and N. P. Bigelow, Phys. Rev. Lett. 103, 250401 (2009).

[72] J.-y. Choi, W. J. Kwon, and Y.-i. Shin, Phys. Rev. Lett. 108, 035301 (2012).

[73] J.-y. Choi, W. J. Kwon, M. Lee, H. Jeong, K. An, and Y.-i. Shin, New J. Phys. 14, 053013 (2012).

[74] L. E. Sadler, J. M. Higbie, S. R. Leslie, M. Vengalattore, and D. M. Stamper-Kurn, Nature (London) 443, 312 (2006).

[75] M. Vengalattore, S. R. Leslie, J. Guzman, and D. M. Stamper-Kurn, Phys. Rev. Lett. 100, 170403 (2008).

[76] J. Kronjäger, C. Becker, P. Soltan-Panahi, K. Bongs, and K. Sengstock, Phys. Rev. Lett. 105, 090402 (2010).

[77] E. M. Bookjans, A. Vinit, and C. Raman, Phys. Rev. Lett. 107, 195306 (2011).

[78] J. Dalibard, F. Gerbier, G. Juzeliūnas, and P. Öhberg, Rev. Mod. Phys. 83, 1523 (2011).

[79] Y.-J. Lin, R. L. Compton, K. Jiménez-García, J. V. Porto, and I. B. Spielman, Nature (London) 462, 628 (2009).

[80] Y.-J. Lin, K. Jiménez-García, and I. B. Spielman, Nature (London) 471, 83 (2011).

[81] C. Pethick and H. Smith, Bose-Einstein Condensation in Dilute Gases (Cambridge University Press, Cambridge, England, 2002).

[82] J. Lovegrove, M. O. Borgh, and J. Ruostekoski (unpublished).

[83] E. G. M. van Kempen, S. J. J. M. F. Kokkelmans, D. J. Heinzen, and B. J. Verhaar, Phys. Rev. Lett. 88, 093201 (2002).
[84] A. Crubellier, O. Dulieu, F. Masnou-Seeuws, M. Elbs, H. Knöckel, and E. Tiemann, Eur. Phys. J. D 6, 211 (1999).

[85] P. W. Anderson and G. Toulouse, Phys. Rev. Lett. 38, 508 (1977).

[86] N. D. Mermin and T.-L. Ho, Phys. Rev. Lett. 36, 594 (1976).

[87] Ü. Parts, J. M. Karimäki, J. H. Koivuniemi, M. Krusius, V. M. H. Ruutu, E. V. Thuneberg, and G. E. Volovik, Phys. Rev. Lett. 75, 3320 (1995).

[88] F. K. Fatemi, K. M. Jones, and P. D. Lett, Phys. Rev. Lett. 85, 4462 (2000).

[89] D. J. Papoular, G. V. Shlyapnikov, and J. Dalibard, Phys. Rev. A 81, 041603 (2010).

[90] S. Burger, K. Bongs, S. Dettmer, W. Ertmer, K. Sengstock, A. Sanpera, G. V. Shlyapnikov, and M. Lewenstein, Phys. Rev. Lett. 83, 5198 (1999).

[91] J. Denschlag, J. E. Simsarian, D. L. Feder, C. W. Clark, L. A. Collins, J. Cubizolles, L. Deng, E. W. Hagley, K. Helmerson, W. P. Reinhardt, S. L. Rolston, B. I. Schneider, and W. D. Phillips, Science 287, 97 (2000).

[92] K.-P. Marzlin, W. Zhang, and E. M. Wright, Phys. Rev. Lett. 79, 4728 (1997).

[93] E. L. Bolda and D. F. Walls, Phys. Lett. A 246, 32 (1998).

[94] J. E. Williams and M. J. Holland, Nature (London) 401, 568 (1999).

[95] T. Isoshima, M. Nakahara, T. Ohmi, and K. Machida, Phys. Rev. A 61, 063610 (2000).

[96] Z. Dutton and J. Ruostekoski, Phys. Rev. Lett. 93, 193602 (2004).

[97] M. R. Matthews, B. P. Anderson, P. C. Haljan, D. S. Hall, C. E. Wieman, and E. A. Cornell, Phys. Rev. Lett. 83, 2498 (1999).

[98] A. E. Leanhardt, A. Görlitz, A. P. Chikkatur, D. Kielpinski, Y. Shin, D. E. Pritchard, and W. Ketterle, Phys. Rev. Lett. 89, 190403 (2002).

[99] Y. Shin, M. Saba, M. Vengalattore, T. A. Pasquini, C. Sanner, A. E. Leanhardt, M. Prentiss, D. E. Pritchard, and W. Ketterle, Phys. Rev. Lett. 93, 160406 (2004).

[100] M. F. Andersen, C. Ryu, P. Cladé, V. Natarajan, A. Vaziri, K. Helmerson, and W. D. Phillips, Phys. Rev. Lett. 97, 170406 (2006).

[101] J. Ruostekoski and Z. Dutton, Phys. Rev. A 72, 063626 (2005).

[102] J. Javanainen and J. Ruostekoski, J. Phys. A 39, L179 (2006).

[103] M. Eto, K. Kasamatsu, M. Nitta, H. Takeuchi, and M. Tsubota, Phys. Rev. A 83, 063603 (2011).

[104] A. S. Schwarz, Nucl. Phys. B 208, 141 (1982).

[105] M. Alford, K. Benson, S. Coleman, J. Marchrussel, and F. Wilczek, Nucl. Phys. B 349, 414 (1991).

[106] M. Greiner, O. Mandel, T. Esslinger, T. W. Hänsch, and I. Bloch, Nature (London) 415, 39 (2002).

[107] R. Jördens, N. Strohmaier, K. Günter, H. Moritz, and T. Esslinger, Nature (London) 455, 204 (2008).

[108] U. Schneider, L. Hackermüller, S. Will, T. Best, I. Bloch, T. A. Costi, R. W. Helmes, D. Rasch, and A. Rosch, Science 322, 1520 (2008).

[109] P. K. Kundu and I. M. Cohen, Fluid Mechanics, 4th ed. (Academic, New York, 2008).

[110] N. Suzuki, H. Takeuchi, K. Kasamatsu, M. Tsubota, and H. Saito, Phys. Rev. A 82, 063604 (2010).

[111] E. Lundh and J.-P. Martikainen, Phys. Rev. A 85, 023628 (2012). 